\title{
Antimicrobial Stewardship Programs: A Review
}

\author{
Akram N. Salah ${ }^{\mathrm{a}}$, Ghadir S. El-Housseiny ${ }^{\mathrm{b}}$, Nooran S. Elleboudy ${ }^{\mathrm{b}}$ Mahmoud A. Yassien ${ }^{\mathrm{b}^{*}}$ \\ ${ }^{a}$ Experimental and Advanced Pharmaceutical Research Unit, Faculty of Pharmacy, Ain Shams University, Cairo \\ 11566, Egypt \\ ${ }^{\mathrm{b}}$ Department of Microbiology and Immunology, Faculty of Pharmacy, Ain Shams University, Cairo 11566, Egypt
}

\begin{abstract}
Antimicrobial resistance is a global crisis that requires urgent action to halt its spread. The rate of deaths due to antibiotic resistance from bacterial infection reached 25,000 annually. Resistance rates to Escherichia coli and Klebsiella pneumoniae have risen to reach $40 \%$ and $56 \%$ respectively, in addition to high resistance rates of Acinetobacter baumannii to fluoroquinolones and other antibiotics that reached $56 \%$. So it has become an urgent need to establish robust programs to control antibiotic resistance and optimize the use of antibiotics in hospitals. In Egypt particularly, the rate of antibiotic resistance becomes high due to wrong traditions of dispensing and administering antibiotics in hospitals and pharmacies, all that drove us to build a program controlling all these obstacles. Antimicrobial stewardship programs (ASPs) are strategic programs to prevent the development of microbial resistance and monitor prescription patterns of clinicians inside hospitals. In addition to minimizing the toxic effects, overuse of antibiotics and improve patient health, and minimize the emergence of antibiotic resistance in the shadow of the lack of development of novel antimicrobial agents. In this review, we aimed to discuss antimicrobial stewardship programs, their core elements, clinical importance, essential requirements, and their implementation in Egypt. In conclusion, the development of national guidelines for antimicrobial stewardship programs would be a useful step to help clinicians in making evidence-based treatment choices regarding antibiotic therapy and the implementation of better antibiotics during pandemics. We hope this review helps the medical staff to perform stewardship programs to control antibiotic resistance in Egyptian healthcare settings.
\end{abstract}

Keywords: Antimicrobial stewardship programs; microbial resistance; antimicrobial agents-hospitals;

core elements.

*Correspondence | Mahmoud A. Yassien; Department of Microbiology and Immunology, Faculty of Pharmacy, Ain Shams University, Cairo 11566, Egypt. Email: mahmoud.yassien@pharma.asu.edu.eg

Citation | Salah AN, El-Housseiny GS, Elleboudy NS Yassien MA, 2021. Antimicrobial Stewardship Programs: A Review. Arch Pharm Sci ASU 5(1): 143-157

DOI: 10.21608 /aps.2021.76105.1059

Print ISSN: 2356-8380. Online ISSN: 2356-8399.

Received 11 May 2021. Accepted 15 June 2021.

Copyright: ${ }^{\circledR} 2021$ Salah et al. This is an open-access article licensed under a Creative Commons Attribution 4.0 International License (CC BY 4.0), which permits unrestricted use, distribution, and reproduction in any medium, provided the original author(s) and source are credited.

Published by: Ain Shams University, Faculty of Pharmacy

\section{INTRODUCTION}

Antimicrobials have transformed the medical practice, making lethal infectious diseases readily treatable in addition to other medical advances, such as organ transplants and chemotherapy against cancer possible [1]. In the past, antibiotics development booming meant that when bacterial resistance developed, newer drugs were always available to cure the emerging resistant bacteria. Around fourteen classes of new antibiotics were discovered between the years 1935 and 2003. But rapid antimicrobials development happens with a cost - antibiotic resistance. In the hospital, resistance to antimicrobial and antifungal agents poses a great concern. In the year 2003, Intensive 
care units (ICUs) informed the Centers for Disease Control and Prevention (CDC) that 60\% of Staphylococcus aureus bacterial strains were resistant to methicillin [2]. Despite decreasing the rate of methicillin-resistant S. aureus (MRSA) invasive infectious disorders related to health care settings in 2010, CDC reported that MRSA strains resistant to glycopeptides (e.g vancomycin) are being less rare $[3,4]$. There has also been an increase in the resistance of Gramnegative bacilli [5]. Programs like the international Study for Monitoring Antimicrobial Resistance Trend (SMART) [6] and the SENTRY Antimicrobial Surveillance Program have reported fundamental increases in the rate of emergence of Klebsiella spp. strains resistant to cephalosporins (third-generation), Pseudomonas spp. producing extended-spectrum $\beta$-lactamase (ESBL), and E.coli and Klebsiella pneumoniae which are resistant to quinolones [7, 8]. Moreover, it was shown that there are high rates of resistance of Gram-negative bacteria that cause a large number of deaths reaching more than 16000 deaths per year as reported by the European Union reported [9].

According to European Centre for Disease Prevention and Control, more than $82.1 \%$ of Salmonella spp. was resistant to different types of antibiotics [10].

In 2021, E. coli clinical isolates obtained from human specimens in community and hospital settings were more resistant to penicillin, tetracyclines, and sulfa drugs with more than $70 \%$ and $40 \%$ increase in resistance to cephalosporins, in addition to its resistance to fluoroquinolones with more than 68\% [11].

Through 50-60 years, these medical science successes were starting to fade as bacteria, viruses, and fungi started to develop microbial resistance to antibiotics. So, the fight against microbial pathogens has been a losing battle despite relentless attempts by scientists to develop novel and more effective antimicrobial agents against pathogens. Although antimicrobial resistance (AMR) is a naturally occurring phenomenon, the process has been much enhanced because of the overuse and misuse of antimicrobial agents in humans. Consistent expansion of AMR has stunted both the treatment and prevention of infections that came from parasites, bacteria, viruses, and fungi [12]. So, AMR makes a progressive grave risk for global and universal public health in the last decades and has now been considered as the highest health risk in the 21 st centenary. In the United States of America, the healthcare cost annually related to AMR was 55\$ billion in 2013, and every year more than two million of the US population are exposed to multiple drug-resistant infections, which need more than 23,000 deaths. The European Center for Disease Prevention and Control (ECDC) and European Medicines Agency (EMEA) jointly estimated that in 2009, the overall annual healthcare productivity and expenditure losses were over $€ 1.5$ billion because of the additional cost to AMR management of the infectious disease [12]. Additionally, hospital costs were estimated to be over $900 €$ million and multidrug-resistant (MDR) microbial infections claimed around 33,000 lives. MDR was concreted as resistance to one antimicrobial agent in three antimicrobial classes or more, extensively-drug-resistant (XDR) was delineated as no sensitivity to one agent at least in all classes but two or fewer antimicrobial agents classes (i.e., bacterial isolates remain sensitive to one category or maximum two categories) and pandrug-resistant (PDR) was described as resistance to all drugs in all antimicrobial classes. Furthermore, many studies reported that patients and pediatric patients' parents were ill-informed about AMR and lacked understanding of how resistance interferes with treatment failure or at least difficulty. This defect in understanding fails to recognize any responsibility for tackling the 
problem of AMR [9].

Microorganisms succumbed to Darwinian selection to make some strict mechanisms to diminish the killing effects of antimicrobial agents. Naturally, most of the antibiotics are produced by microbes like environmental fungi or saprophytic bacteria, others are modified synthetic antibiotics, whereas a few are completely synthetic like sulphonamides and fluoroquinolones. Various organisms evolved defensive phenomena against them such as inhibition of drug entry or distribution, alterations in the target site, enzyme production that may break the antimicrobial agents down, and efflux pump overexpression. For that cause, AMR may simply depict the Darwinian competition from natural microbial-derived antimicrobials. The functional metagenomic analysis of soil microbes has revealed diversity in genetic materials associated with AMR. Although little has been reported in human bacteria about this aspect, the production of enzymes (such as $\beta$-lactamases) is an example except for a naturally-making present bacterial resistance mechanism and has an influence on human health [13].

From 2005 till 2018 the rate of resistance of Acinetobacter baumannii to different carbapenems antibiotics has increased by approximately two-fold in addition to its resistance to different antibiotics classes like fluoroquinolones, macrolides, and cephalosporins which has reached $80 \%$ in some countries and $56 \%$ in others which is considered a great disaster [14].

Infections caused by antibiotic-resistant bacteria are readily associated with increased costs and morbidity andlor mortality rates [15]. Even though antibiotic-resistant infections and the associated healthcare costs have been more completely described in many developed countries, bacterial resistance is problematic worldwide [16]. Medical experts have declared that we are near to the age of no effective antimicrobial agents as the peak of the antimicrobial resistance challenge is approaching [17]. Optimizing antibiotics use has become so critical to treat microbial infections effectively, prevent patients from harmful events caused by unsuitable use of antibiotics, and control antibiotic resistance. Antibiotic Stewardship Programs (ASPs) can strongly help clinicians develop clinical outcomes and decrease harm by improving antibiotic prescription [18]. ASPs in hospitals can raise infection treatment rates while reducing treatment failures, adverse reactions, antibiotic resistance, hospital, and healthcare costs and stay durations, and Clostridium difficile infections [19]. Antimicrobial stewardship program is defined by the Infectious Diseases Society of America (IDSA) as a "rational and systematic approach to antimicrobial agents use in to achieve optimal outcomes" [20].

ASP team members should be trustable and with medical background and willing to work with others as part of ASP, the team may be composed of two to three members or up to six or more for larger facilities [21, 22]. ASP activities have traditionally been established exclusively by a pharmacist with oversight from an infectious disease physician. But the last decade has shown a dramatic elevation in rapid diagnostic microbiological technologies offering opportunities for ASP [23].

\section{ASP clinical importance and goals}

ASP has been described as "the optimum selection, dose, and antimicrobial cure duration that leads to the top of clinical outcome for prevention and complete infection treatment, with minimum toxicity level to the patient and minimum negative impact on sequent antibiotic resistance" [24]. The ASPs have three goals. The first goal is to work with all health care practitioners for helping each patient get the most appropriate antimicrobial agent with the optimum 
and correct dosage and duration of treatment. Joseph and Rodvold [25] have described "4 D's of the optimum antimicrobial agents' therapy" as the: right Dose, right Drug, De-escalation to microbe-directed therapy, and finally right Duration of treatment. The good care of a patient with infection means cure with the right, dosed antibiotic with the least collateral damage (ie, development of resistance in the patient or one of their the his her contacts). An extra benefit of these programs that target to optimize the use of antibiotics is that they generally get cost savings because not many doses of antibiotics are consumed, and relatively cheap antimicrobials are selected. Comprehensive programs declared savings between $\$ 200,000$ and $\$ 900,000$ per year when ASPs are applied [26].

The second goal is to forbid the misuse of antimicrobial agents in addition to overuse, and abuse [27]. In hospitals and outpatient settings, antibiotics may be prescribed when they are not needed. Self-medication and antibiotics selfdispensing is a common practice in developing countries, including Egypt [28]. Antibiotics may be prescribed to treat viral infections or noninfectious processes like febrile patients with acute pancreatitis or small dermal abscesses that will resolve with drainage and incision. Antibiotics are also misused frequently, like in the common matter of using broader in-spectrum antibiotics to treat community-acquired infections or prescribing antibiotics not based on culture and sensitivity tests. Antibiotics misuse is difficult to define, but it might be described as the use of a particular antibiotic over another for financial or marketing interest.

The third goal of ASPs is to decrease the emergence of resistance, both at the community level and the individual patient level since antibiotic use changes susceptibility patterns. Patients who are exposed to antimicrobials are at higher risk of being infected or colonized by drug-resistant organisms [29]. The most common reason for Clostridium difficile pseudomembranous colitis diarrhea development is exposure to antimicrobials. The Gram-negative bacterial resistance to cephalosporins and carbapenems had been increased 10 to 12 -fold with exposure to broad-spectrum antimicrobial agents [30].

\section{Planning for ASP}

ASPs in healthcare facilities must not be vertical, they must cut across other programs to optimize the use of antibiotics, and to improve the quality of infection management.

To plan for an ASP, health-care facilities can be differentiated as shown in Fig. 1.

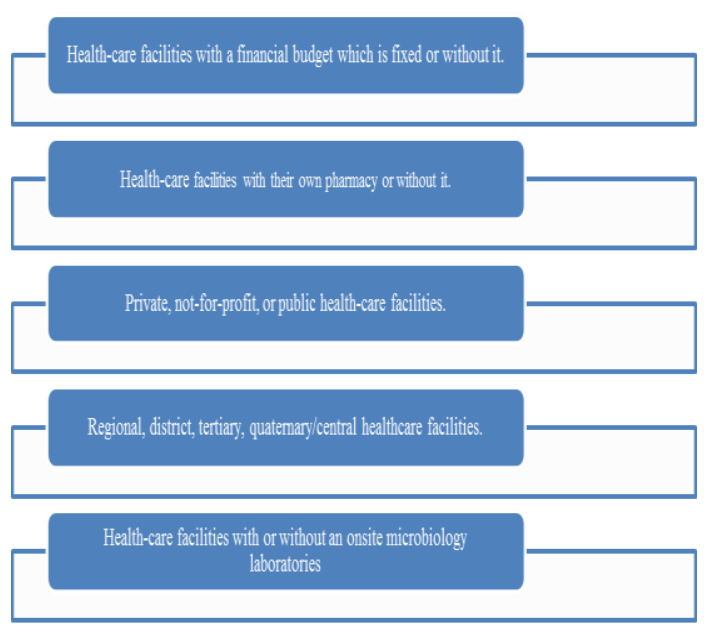

Fig.1. Differentiation of health-care facilities [31]

An ASP program in a tertiary large hospital with various specialties will be larger and more complex than the program in a district hospital. Therefore, healthcare facility management and a stewardship committee and/or ASP team must decide which strategies are the best fit and most compliant to their local setting, depending on situational analysis and an action plan development. The ASP implementation is a stepwise process working dynamically with each facility established in the hospital [32]. 
A multidisciplinary ASP team of various healthcare professionals should be established, who possess the competencies collectively and take functions to implement and deliver the ASP program successfully for healthcare facilities. Ideally, the ASP team should comprise a prescriber (clinician), a nurse, a pharmacist, and a microbiologist or laboratory technician. If available, an infectious disease doctor and a pharmacologist are also recommended. The staff (who were nominated) in the team then need a dedicated time to successfully implement the program, and their ASP must be in their job description and performance contract [33].

The role of the physician is to review hospital cases with the pharmacists in a regular form, from once per week up to five times weekly. The pharmacist should pre-review cases for medical discussion to cause a maximum efficient use of antibiotics [34]. In addition to the physician and pharmacist role, we cannot neglect the nurse's role in ASP by initiating an antibiotic timeout and questioning the medical necessity of cultures or encouraging the prompt transition from intravenous to oral antibiotics in addition to recording an accurate drug allergy history like penicillin hypersensitivity and finally ensuring suitable techniques when collecting the specimen for culture [35]. A clinical Microbiologist (who would be a pharmacist too) is important for implementing a complete ASP by performing and releasing a full antibiogram for the hospital which lists the micro-organisms that are susceptible to the hospital's formulary antibiotics over a given time frame and usually gives a picture of the susceptibility of clinical isolates submitted to the microbiology laboratory during a year and reflects bacterial isolates collected from the entire hospital. This gives guidance for empiric antimicrobial therapy in a setting where rates of microbial resistance are high for expected organisms and monitors changes in resistance over time, so it helps an ASP in its formulary decisions [36].

\section{ASP essential requirements}

There are four essential requirements for establishing ASP in hospitals as the main substrates to start it [37].

The four essential requirements are antibiograms, electronic system records, updated infection control reports, and research committee, and we will discuss each one in detail.

\subsection{Antibiograms}

An antibiogram (ABG) is a summary of the cumulative susceptibility (sensitivity) of clinical bacterial isolates in a certain hospital or region to formulary antibiotics. It is used mainly to guide the choice of empirical therapy and track resistance patterns helping ASP to start and follow its success [38]. The antibiogram itself must be well designed to reflect changes in hospital antimicrobial drug use and give periodical reports according to updated antibiotic use and consumption [39]. Earlier research has shown that the use of the ABG to direct the selection of cautious antibiotics was less than required. Use by non-infectious disorder practitioners had been limited, and antibiotic prescribing had not aligned with stewardship goals. Later research recommends increasing the utility of antibiograms through stratification, education, and cross-tabulation of susceptibility data for combination therapy of antibiotics. These approaches focus on the rising use of the cumulative and updated antibiograms and emphasizing that the data influence the antibiotic selected for therapy. They predicted that these antibiograms will be influential methods for increasing the use of relatively narrow spectrum empirical antibiotic regimens [40].

\subsection{Electronic system record}

In many countries, healthcare systems have 
been changed predominantly in terms of the electronic medical record (EMR) [41]. Now each hospital must have an EMR which is a part of joint commission international (JCI) requirements to save all patients' data and track any changes in patient's treatment or diseases. EMR can also be adjusted to track the implementation of ASP recommendations, by what and to who were those ASP recommendations communicated, saving and documenting whether the recommendations were followed, and recording any impacts on antimicrobial days of therapy [42].

\subsection{Updated infection control reports}

The Society for Healthcare Epidemiology of America (SHEA) and the Association for Professionals in Infection Control and Epidemiology (APIC) made highlights the importance of infection preventionists (IPs) and healthcare epidemiologists (HEs) ineffective ASPs [43].

Keeping infection control reports and updating them on monthly basis is one of the cost-effective interventions against antimicrobial resistance [44] and is important to reduce the spread of antibiotic resistance in the presence of excessive rates of healthcare-associated infections (HAIs) [45]. Reporting the rates of MRSA, vancomycin-resistant Enterococcus (VRE), MDR pathogens, and $\mathrm{Cl}$. difficile pathogenicity is one of the main functions of infection control as it gives complete data about recurrent infections in the hospital and causative microorganisms and how to eradicate them, in addition to performing sterilization procedures and assessing the choice, concentration, and effectiveness of the used antiseptics and disinfectants [46, 47].

\subsection{Research committee}

Assigning a research committee in each hospital responsible for making updated studies about resistance patterns within the hospital and ways to solve them and being an advisory committee for all ASP teams will greatly improve the ASP functions and elements [48].

\section{The core elements of the ASP}

As mentioned previously, optimizing the antibiotics usage is critical to infection treatment, prevent patients from harmful events caused by superfluous antibiotic consumption, and concise antibiotic resistance. ASPs can help physicians to ameliorate clinical outcomes then reduce harm by prescribing the appropriate antibiotic.

In 2019, CDC declared the updates of the hospital core elements to summarize all lessons obtained from five years of experience and novel evidence from the stewardship field as shown in the figure and their details listed below the Fig. 2 [49].

\subsection{Accountability}

The appointment of leaders or co-leaders like a physician orland pharmacist, who is responsible for program management with outcomes. Then the role of Pharmacy Expertise (Drug Expertise), ideally as the co-leader of this program, is to assist in leading efforts of implementation to improve the use of antibiotics.

\subsection{Action}

Implement many interventions, like prospective audit and preauthorization or feedback, for the same target, improving antibiotic use.

\subsection{Tracking}

That occurs by monitoring antibiotic prescribing and the impact of interventions, in addition to other important outcomes of bacterial resistance patterns (e.g Cl. difficile). 


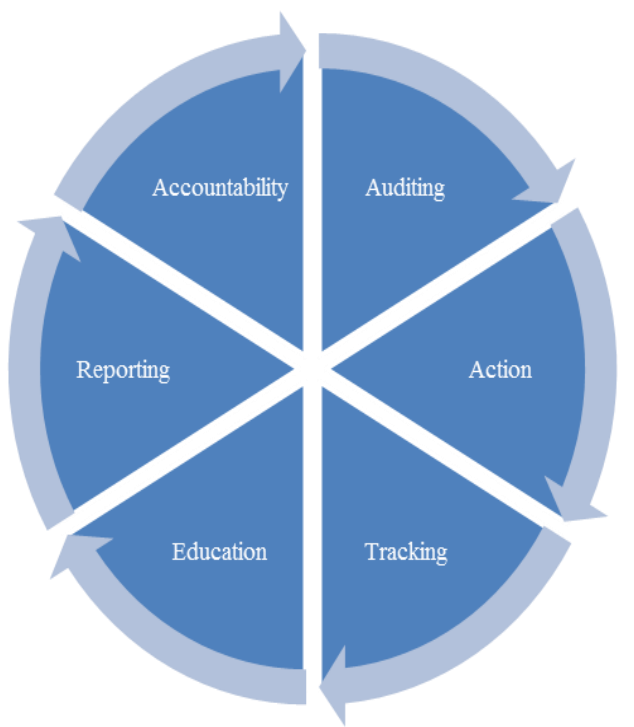

Fig. 2. The core elements of antimicrobial stewardship program

\subsection{Reporting}

Reporting information on the use of antibiotics, consumption, prescribing, and resistance to physicians, pharmacists, nurses, hospital leadership.

\subsection{Education}

It is important to educate clinicians, prescribers, nurses, pharmacists, and patients also regarding adverse effects and reactions from antibiotics use, antibiotic resistance, and optimal prescribed dosage and administration.

\subsection{Auditing}

Prospective auditing and feedback are not a novel concept, in the past decades; it was called immediate concurrent feedback. The prospective audit team comprises a physician (especially an infectious diseases physician or a clinical microbiologist) and clinical pharmacists [50]. They are responsible for monitoring and tracking any problems as well as following up the fulfillment of ASP protocols and guidelines.

\section{Steps for improving the ASP}

The following four steps may help ease suitable antibiotic use and decrease the rise of multi-resistant pathogens and other microorganisms, like medication-resistant fungi [51].

\subsection{Step 1: Reward antimicrobial stewardship}

A physician can share by giving a reward of network health systems that have a documented ASP and manifesting outcomes improvement. Physicians can monitor participating systems bacterial rate of resistance and overall antimicrobial use as performance endpoints. Rewards could include improved preferred network status or reimbursement rates $[\mathbf{5 3}, \mathbf{5 3}]$.

\subsection{Step 2: Leverage quality scores}

There are three measures to assess the quality of ASP including:

Avoiding antibiotic treatment in adult patients with acute bronchitis (AB) [54].

Appropriate testing for pediatrics with pharyngitis [55].

Appropriate cure for pediatrics with upper respiratory tract infection (URTI) [56].

Performance on every single measure 
develops with suitable antibiotic use. Performance evaluating on the Plan All-Cause Readmissions (PACR) quality measure can be beneficial as well, considering this microbial infection is a reason for re-hospitalization. Physicians must routinely evaluate the quality scores on these measurements and also identify practices, prescribers, or hospitals that may be rebounding to suboptimal performance. The physician might notify providers of poor performance, reinforce the ASP importance, and give guidance on how to improve. Repeated poor performers might be subject to penalties, including exclusion from preferable high- performance networks.

The National Committee for Quality Assurance (NCQA) included in HEDIS 2017 a new measure, the Standardized HealthcareAssociated Infection Ratio. This measure is engaged to evaluate a potential infection risk for hospitals where members are admitted to within the plan's networks.

The four healthcare-associated infections (Nosocomial infections) are (reported by Centers for Medicare and Medicaid Services "CMS") are shown in Fig. 3.

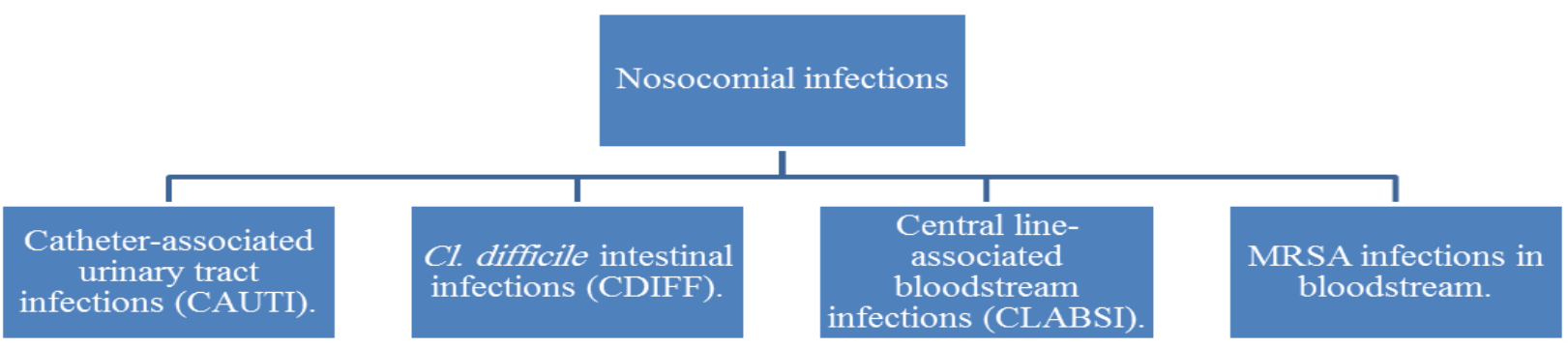

Fig. 3. The four healthcare-associated infections are (reported by Centers for Medicare and Medicaid Services "CMS")[57]

Physicians also monitor stewardship program performance on the new assessment as data is available and can determine facilities contributing to a sub-optimal level of performance. Physicians may need action plans for improving the performance from the facilities, many of which are subject, as of January 2017, to The Joint Commission's new Antimicrobial Stewardship Standard (JAASS). Hospital facilities performing well on this measure or presenting improvement could be rewarded as noted previously. Data of performance on healthcare-acquired (nosocomial) infections between Medicare-certified hospitals are available through hospital comparing and other quality metric and performance aggregator tools like Qnav. Plans may have a target of reviewing high-volume hospitals' performance within their network and improve strategic collaborations proactively on decreasing infections and antimicrobial stewardship initiatives. Collaborations could comprise health systems in addition to many other stakeholders with high potentials, such as employer coalitions, pharmaceutical companies [58].

\subsection{Step 3: Implement appropriate controls}

Physicians may also utilize prior authorization programs when the goal is controlling and optimizing inappropriate antibiotic use. But unfortunately, prior authorization for antibiotics is problematic as antimicrobials agents tend to be cheap, making administration of the program costly as ASP's main aim is to reduce the financial costs associated with inappropriate use of antibiotics [59].

Additionally, this strategy necessitates having a medical expert to ensure empirical antibiotics' appropriateness. However, culture sensitivity and 
susceptibility results and other many diagnostic results will be available over the next two to three days making a chance to revisit the therapy actions [60]. So, the restriction leads to an increase in the bacterial resistance. An example of this was in neonatal ICU where Reed et al. reported that they tried to perform a ceftazidime restriction policy to decrease the colonization rate with ceftazidime-resistant Gram-negative bacila li [61]. This policy led to $96 \%$ decreasing in ce use but there was actually a petty raise in the ceftazidime-resistant microorganisms' density (1.57 to 2.16 clinical isolates/100 patient-days).

Quantity limits can facilitate appropriate regimens while limiting daily doses and excessive therapy duration. ASP could apply rules that allow patients newly discharged from the hospital or with lab results to get immediate approval of appropriate therapy. Physicians can also use retrospective drug utilization reviews and reports to identify prescribers who write excessive amounts of antimicrobial agent prescriptions or prescribe antibiotics for inappropriate indications. Physicians should communicate the issues with these clinicians and outline ways to improve the performance. Finally, physicians may introduce an antimicrobial stewardship program through existing pharmacies and population health management programs. Many chronic conditions are completely managed using multiple interventions (e.g., immunizations, medication adherence, referrals, depression screenings, a transition of care). Appropriate patient antimicrobial management could be presented through the care management process [62].

\subsection{Step 4: Patient education campaign}

There are many programs worldwide to explain why antibiotics should not be administrated for many illnesses, prescribing recommendations and guidelines for physicians, social media platforms, computerized decision aids, mobile applications, and courses for medical staff professionals [63].

Physicians have accessibility and the chance to educate a broad population. A physician can implement a public education in the form of a campaign for communicating with the population and counseling them on the threat of antibiotic resistance. After these detailed steps, physicians can practice antimicrobial stewardship program in a way that limits negative impact on patients and raise their satisfaction level in addition to their health [51].

\section{The Pandemic stewardship program for COVID-19 and Implementing ASPs in tertiary care settings of the low-and middle-income country as a general aspect and in Egypt in particular}

The antibiotic misuse and resistance problem appear in Egypt strongly. In May 2018 the World Health Organization (WHO) Egypt country office is supporting the Egypt government in antibiotic resistance national action plan drafting. An antibiotic resistance coordination committee has been made within the Ministry of Health and Population, comprising representatives from various departments such as infection prevention and control, surveillance, central administration of pharmaceutical affairs (CAPA), central public health laboratories, quality control (QC), environmental health section, training, development and research, the national information center and the curative section [64].

Egypt assessed the ASP according to CAPA in April 2017 as they set a global action plan by setting up governance structures, surveillance system to optimize a regular antimicrobial use and consumption, reporting an intervention for rational use of antimicrobials, track and measure the progress of the national ASP (NASP), and conduct to awareness campaigns for patients and doctors awareness [65]. 
Starting for prevention and infection control units in hospitals to control microbial growth and resistance through the antibiotics use optimization in hospitals and auditing to perform these programs comes from the ministry of health and CAPA.

Reaching that the proper antibiotic usage entails the alignment of adequate knowledge, proper practice, and a positive manner.

This program is applied successfully in some of Al Zagazig hospitals under the supervision of the hospital ASP team [66].

The dissemination and prevalence of Corona Virus Disease-19 (COVID-19) around the world has been followed by raising antibiotics consumption. This is regarding bacteria superinfection in COVID-19 patients. The pathogens eradication is challenging in countries with low or middle income (e.g Egypt) because there are no available and cost-effective readily biological or clinical laboratory markers that can effectively distinguish between viral and bacterial infections. Fortunately, the threat of COVID-19 spread, we could face it, as there has been a promising awareness of the ASPs importance to control this antibiotics misuse and optimize the antibiotics consumption during the COVID storm. The initial observation of high antibiotic consumption during this pandemic viral was influenced by early reports from China (Wuhan) indicating that more than $49 \%$ of patients who died from coronavirus had a 2ry bacterial infection [67].

\section{Other supplemental strategies for ASPs development}

\subsection{Conducting a prospective audit with intervention and feedback}

Before the ASP implementation, the inpatient pharmacist performed a general review of antibiotic orders to detect any discrepancies with individual antimicrobials, including therapeutic indication, dose, frequency (hepatic and renal function), and duration of therapy (DOT). Since the inception of the ASP, the pharmacist nowadays reviews each medication order (prescribed in the medications sheet) by physically reviewing every patient's clinical chart. These reviews include antibiotics given by the intravenous route, with particular attention to $3^{\text {rd }}$ generation cephalosporins; ceftazidime, meropenem, ciprofloxacin, piperacillin/tazobactam, teicoplanin, and vancomycin. If an order is found to be suboptimal or inappropriate, the pharmacist contacts the prescriber before the medication dispenses an effort to adjust therapy to an antimicrobial agent that is more specific and individualized to each patient case [68].

\subsection{Enforce drug formulary restriction and need for authorization for non-formulary antibiotics (NFA)}

The formulary restriction and preauthorization criteria for antibiotics are considered to form the most effective approaches to control the antimicrobial agent use, formulary-related decisions are made by the internal pharmacy. Formulary restriction refers to limiting a facility's antimicrobial agent formulary according to many factors such as efficacy, toxicity, redundancy, and cost. For example, linezolid, an antibiotic prescribed for vancomycin-resistant enterococci (VRE) and multidrug-resistant bacterial strains (Staphylococcus aureus), is not available on the hospital's formulary but can be ordered as a caseby-case. If a non-formulary antibiotic is prescribed, the prescriber (physician) must provide a rationale (justification) for its use, and the drug must be authorized by the ASP pharmacist [69].

\section{Conclusion}

In conclusion, a lack of control on antibiotic 
use in hospitals will inevitably lead to a health disaster. Providing ASPs regarding antibiotic use and prescription indications and the consequences of misuse or overuse and inappropriate administration is urgently needed. The dissemination and development of national guidelines for antimicrobial stewardship programs would be a useful and remarkable step to help clinicians in making evidence-based treatment choices regarding antibiotic therapy and implementation of better antibiotic use in hospitals.

\section{Declarations}

\section{Ethics approval and consent to participate}

Not available

\section{Consent to publish}

Not available

\section{Availability of data and materials}

Not available

\section{Competing interests}

The authors declare that they have no competing interests.

\section{Funding statement}

This research did not receive any specific grant from funding agencies in the public, commercial, or not-for-profit sectors.

\section{Authors' contributions}

ANS has collected the data for the manuscript under the supervision and guidance of MAY, NSE, and GSE. ANS, NSE, and GSE have written the first draft of the manuscript. GSE and NSE have helped in writing and revising this manuscript. All authors have read and approved the final manuscript.

\section{REFERENCES}

1. Jump RLP, Gaur S, Katz MJ, Morgan J Katz, Christopher J Crnich, Ghinwa
Dumyati, Muhammad S Ashraf, Elizabeth Frentzel, Steven J Schweon, Philip Sloane, and David Nace. Template for an Antibiotic Stewardship Policy for Post-Acute and Long-Term Care Settings. J Am Med Dir Assoc 2017; 18(11):913-20. DOI: 10.1016/j.jamda.2017.07.018

2. CDC. System N. Division of Healthcare Quality Promotion, National Center for Infectious Diseases, Centers for Disease Control and Prevention, Public Health Service US. Available at: https://www.cdc.gov/ncezid/dhqp/index.html 2003.

3. Kallen AJ, Mu Y, Bulens S. Healthcare-associated invasive MRSA infections. JAMA 2010; 304(6):641-8. DOI:10.1001/jama.2010.1115

4. Investigation and control of vancomycinintermediate and -resistant Staphylococcus aureus (VISA/VRSA): a guide for health departments and infection control personnel. Available at: http://www.cdcgov/ncidod/dhqp/pdf/ar/visa vrsa_guide 2011.

5. Kallen AJ, Srinivasan A. Current epidemiology of multidrug-resistant gram-negative bacilli in the United States. Infect Control Hosp Epidemiol 2010; 31(1):51-4. DOI:10.1086/655996.

6. Rossi F, Baquero F, Po-Ren Hsueh, David L Paterson, Grant V Bochicchio, Theresa A Snyder, Vilas Satishchandran, Kathleen McCarroll, Mark J DiNubile, and Joseph W Chow. In vitro susceptibilities of aerobic and facultatively anaerobic Gram-negative bacilli isolated from patients with intra-abdominal infections worldwide: 2004 results from SMART (Study for Monitoring Antimicrobial Resistance Trends). J Antimicrob Chemother 2006; 58(1):205-10. DOI: 10.1093/jac/dk1199

7. Fridken SK. Increasing prevalence of antimicrobial resistance in intensive care units. Crit Care Med 2001; 29(4):64-8. DOI: 10.1097/00003246200104001-00002.

8. Doron S, Davidson LE. Antimicrobial stewardship. Mayo Clin Proc 2011: 86(11):1113-23. DOI: 10.4065/mcp.2011.0358.

9. KawtherAabed, NadineMoubayed, and 
SalehaAlzahrani. Antimicrobial resistance patterns among different Escherichia coli isolates in the Kingdom of Saudi Arabia Saudi. J. Biol. Sci 2021.

10. European Centre for Disease Prevention and Control. Resistance levels are still high in bacteria causing foodborne infections. Available at https://www.efsa.europa.eu/en/news/resistancelevels-still-high-bacteria-causing-foodborneinfections 2021.

11. Emmanuel Nji, Joseph Kazibwe, Thomas Hambridge, Carolyn Alia Joko, Amma Larbi, Lois Afua Okyerewaa Damptey, Nana Adoma NkansaGyamfi . High prevalence of antibiotic resistance in commensal Escherichia coli from healthy human sources in community settings. Sci. Rep 2021; 11:3372.

12. Sameer Dhingra, Nor Azlina A. Rahman, Ed Peile, Motiur Rahman, Massimo Sartelli, Mohamed Azmi Hassali, Tariqul Islam, Salequl Islam, and Mainul Haque. Microbial Resistance Movements: An Overview of Global Public Health Threats Posed by Antimicrobial Resistance, and How Best to Counter. Public Health Front. 2020; 8: 535668. DOI: $10.3389 /$ fpubh.2020.535668

13. Bilal Aslam, Wei Wang, Muhammad Imran Arshad, Mohsin Khurshid, Saima Muzammil, Muhammad Hidayat Rasool, Muhammad Atif Nisar, Ruman Farooq Alvi, Muhammad Aamir Aslam, Muhammad Usman Qamar, Muhammad Khalid Farooq Salamat, and Zulqarnain Baloch. Antibiotic resistance: a rundown of a global crisis. Infect Drug Resis2018; 11: 1645-1658. DOI: $10.2147 /$ IDR.S173867

14. Xiaohong Shi, Hong Wang, Xin Wang, Huaiqi Jing, Ran Duan, Shuai Qin, Dongyue Lv, Yufeng Fan, Zhenzhou Huang, Kyle Stirling, Lei Zhang, and Jiazheng Wang. Molecular characterization and antibiotic resistance of Acinetobacter baumannii in cerebrospinal fluid and blood. Plos one $2021 ; 16(2)$. DOI.org/10.1371/journal.pone.0247418.

15. Borg MA, Tiemersma E, Scicluna E, N van de Sande-Bruinsma, $\mathrm{M}$ de Kraker, J Monen, and $\mathrm{H}$ Grundmann. Prevalence of penicillin and erythromycin resistance among invasive
Streptococcus pneumoniae isolates reported by laboratories in the southern and eastern Mediterranean region. Clin Microbiol Infect 2009;15(3):232-7. DOI: $\quad 10.1111 / j .1469-$ 0691.2008.02651.x.

16. Dooling KL, Kandeel A, Hicks LA, Waleed ElShoubary, Khaled Fawzi, Yasser Kandeel, Ahmad Etman, Anna Leena Lohiniva, and Maha Talaat. Understanding Antibiotic Use in Minya District, Egypt: Physician and Pharmacist Prescribing and the Factors Influencing Their Practices. Antibiotics (Basel) 2014; 3(2):233-43. DOI: 10.3390/antibiotics3020233.

17. Majumder MAA, Rahman S. Antimicrobial Stewardship: Fighting Antimicrobial Resistance and Protecting Global Public Health. Infect Drug Resist. 2020;13:4713-38.

18. Davey P, Marwick CA, Claire L Scott, Esmita Charani, Kirsty McNeil, Erwin Brown, Ian M Gould, Craig R Ramsay, Susan Michie. Interventions to improve antibiotic prescribing practices for hospital inpatients. Cochrane Database Syst Rev 2017; 9;2

19. Baur D, Gladstone PB, Francesco Burkert, Elena Carrara, Federico Foschi, Stefanie Döbele, and Evelina Tacconelli. Effect of antibiotic stewardship on the incidence of infection and colonization with antibiotic-resistant bacteria and Clostridium difficile infection: A systematic review and metaanalysis. Lancet Infect Dis 2017; 17(9).

20. Dellit TO, RC McGowan. Infectious Diseases Society of America; Society for Healthcare Epidemiology of America. Clin Infect Dis 2007; 44:159-77. DOI: $10.1086 / 510393$.

21. Jackson S. Long-Term Care Facility Antimicrobial Stewardship Program Start-Up Toolkit. Topeka: Kansas; 2019.

22. Leuthner KD, Doern GV. Antimicrobial stewardship programs. J Clin Microbiol 2013; 51(12):3916-20.

23. Bauer KA, Perez KK, Graeme N Forrest, Debra A Goff. Review of rapid diagnostic tests used by antimicrobial stewardship programs. Clin Infect Dis 2014; 59:3:134-45. 
24. Gerding DN. The search for good antimicrobial stewardship. Jt Comm J Qual Improv 2001; 27(8):403-4. DOI: 10.1016/s1070-3241(01)270345

25. Joseph J, Rodvold KA. The role of carbapenems in the treatment of severe nosocomial respiratory tract infections. EXPERT OPIN PHARMACO 2008; 9(4):561-75.

26. Lutters $\mathrm{M}$, Harbarth $\mathrm{S}$, et al. Effect of a Comprehensive, Multidisciplinary, Educational Program on the Use of Antibiotics in a Geriatric University Hospital. J Am Geriatr Soc

2004; 52(1):112-6.

27. HAMILTON-MILLERD J. Use and abuse of antibiotics. Br J Clin Pharmac 1984;1 8:469-74.

28. Kasim K. Self Medication Problem in Egypt: A Review of Current and Future Perspective. j. curr 2018.

29. Barbosaa TM, Levyab SB. The impact of antibiotic use on resistance development and persistence. Drug Resist. Update 2000; 3(5):303-11. DOI: 10.1054/drup.2000.0167.

30. Patel G, Huprikar S, Stephanie H Factor, Stephen G Jenkins, David P Calfee. Outcomes of carbapenem-resistant Klebsiella pneumoniae infection and the impact of antimicrobial and adjunctive therapies. Infect Control Hosp Epidemiol 2008; 29(12):1099-106.

31. CDC. Antimicrobial stewardship programs in health-care facilities in low- and middle-income countries: a WHO practical toolkit, Geneva 2019.

32. Mendelson M, Morris AM, K Thursky, C Pulcini. How to start an antimicrobial stewardship program in a hospital. Clin Microbiol Infect 2020; 26(4):447-53. DOI: 10.1016/j.cmi.2019.08.007

33. Colligan P, Beggs J. Anthropological and socioeconomic factors contributing to global antimicrobial resistance: a univariate and multivariable analysis. Lancet Planet Health 2018; 2:398-405.

34. Tamar F Barlam EC, Sarah A Zieminski, Sarah A Zieminski, Tsega M Meshesha, Kathryn E Jones, Jorie M Butler, Laura J Damschroder, Matthew
Bidwell Goetz, Karl Madaras-Kelly, Caitlin M Reardon, Matthew H Samore, Jincheng Shen, Edward Stenehjem, Yue Zhang, Mari-Lynn Drainoni. Perspectives of Physician and Pharmacist Stewards on Successful Antibiotic Stewardship Program Implementation: A Qualitative Study. Open Forum Infect. Dis 2020.

35. Carter EJ, Greendyke WG, E Yoko Furuya, Arjun Srinivasan, Alexa N Shelley, Aditi Bothra, Lisa Saiman, and Elaine L Larson. Exploring the nurses' role in antibiotic stewardship: A multisite qualitative study of nurses and infection preventionists. Am J Infect Control 2018; 46(5):492-7. DOI: 10.1016/j.ajic.2017.12.016

36. Schulz LT, Fox BC. Can the Antibiogram Be Used to Assess Microbiologic Outcomes After Antimicrobial Stewardship Interventions? A Critical Review of the Literature. Pharmacotherapy 2012; 32(8):668-76. DOI: $10.1002 /$ j.18759114.2012.01163.x

37. Maria-Stephanie A. Tolg, David M. Dosa. Antimicrobial Stewardship in Long-Term Care Facilities: Approaches to Creating an Antibiogram when Few Bacterial Isolates Are Cultured Annually. J Am Med Dir Assoc 2019; 19(9): 744747. DOI: 10.1016/j.jamda.2018.05.006

38. Ontario PH. Antimicrobial Stewardship Strategy: Antibiograms. Ontario; 2008; 1-17.

39. Schulz LT, Fox BC, Ron E Polk. Can the antibiogram be used to assess microbiologic outcomes after antimicrobial stewardship interventions? A critical review of the literature. Pharmacotherapy 2012; 32(8):668-76.

40. Resman F. Antimicrobial stewardship programs; a two-part narrative review of step-wise design and issues of controversy Part I: step-wise design of an antimicrobial stewardship program. Ther Adv Infect Dis 2020. DOI: 10.1177/2049936120933187

41. Ain AN, Azhim MZ, Wan Puteh SE. An Overview of Electronic Medical Record Implementation in Healthcare System: Lesson to Learn. World Appl. Sci. J. 2013; 25(2):323-32.

42. Cortes-Penfield NW. Automated EMR tool improves antimicrobial stewardship. Infectious 
Diseases News. Available at: https://www.healio.com/news/infectiousdisease/20190808/automated-emr-tool-improvesantimicrobial-stewardship 2019.

43. Manning ML, Septimus EJ. Antimicrobial stewardship and infection prevention-leveraging the synergy: A position paper update. Am J Infect Control 2018; 46(4):364-8. DOI: 10.1016/j.ajic.2018.01.001

44. Lacotte Y, Ardal C, and Marie-Cécile Ploy. Infection prevention and control research priorities: what do we need to combat healthcareassociated infections and antimicrobial resistance? Results of a narrative literature review and survey analysis. Antimicrob Resist Infect Control 2020; 9(1):142.

45. Gilbert GL, Kerridge I. Hospital Infection Prevention and Control (IPC) and Antimicrobial Stewardship (AMS): Dual Strategies to Reduce Antibiotic Resistance (ABR) in Hospitals: Ethics and Drug Resistance: Collective Responsibility for Global Public Health 2020.

46. Pogorzelska M, Stone PW. Certification in infection control matters: Impact of infection control department characteristics and policies on rates of multidrug-resistant infections. Am J Infect Control 2012; 40(2):96-101. DOi: 10.1016/j.ajic.2011.10.002

47. Rutala WA, Weber DJ. Infection control: the role of disinfection and sterilization. J Hosp Infect 1999; 43:43-55.

48. Henderson A, Brouse J. Development of a research committee at a community hospital. Can J Nurs Adm 1992; 5(1):17-9.

49. Atlanta G. CDC. Core Elements of Hospital Antibiotic Stewardship Programs. Available at: https://www.cdc.gov/antibiotic-use/coreelements/hospital.html 2019.

50. McGowan JE. Antimicrobial stewardship-the state of the art in 2011: focus on outcome and methods. Infect Control Hosp Epidemiol 2012; 33:331-7. DOI: $10.1086 / 664755$.

51. Schafer J. Four steps managed care can take to promote antimicrobial stewardship. Healthc Exec 2017.

52. Schafer J. The Role of Payers in Antibiotic Stewardship: Facilitating Transitions of Care for Patients With Active Infection. Int. J. Care Pathw 2017; 3(2):27-8.

53. Haefner M. 4 steps to ensure your antimicrobial stewardship program is evidence-based. clinical leadership and infection control Becker's 2017.

54. Frisina P, Pletcher J. ANTIBIOTIC STEWARDSHIP FOR ACUTE BRONCHITIS: A QUALITY IMPROVEMENT (QI) STUDY IN UNIVERSITY HEALTHCARE. BMJ Qual. Saf 2016; 25(12).

55. Brennan-Krohn T, Ozonoff A. Adherence to guidelines for testing and treatment of children with pharyngitis: a retrospective study. BMC Pediatr. 2018; 18(1):43.

56. Teoh L, Sloan AJ, Michael J McCullough, and Wendy Thompson. Measuring Antibiotic Stewardship Programmes and Initiatives: An Umbrella Review in Primary Care Medicine and a Systematic Review of Dentistry. Antibiotics (Basel) 2020; $\quad 9(9) . \quad$ DOI: 10.3390/antibiotics 9090607

57. Haque M, Sartelli M, et al. Healthcare-associated infections - an overview. Infect Drug Resist 2018; 11:2321-33.

58. Tahoon MA, Khalil MM. The effect of educational intervention on healthcare providers' knowledge, attitude, \& practice towards antimicrobial stewardship program at, National Liver Institute, Egypt. Egypt. Liver J 2020; 5.

59. Chung GW, Wu JE. Antimicrobial stewardship: a review of prospective audit and feedback systems and an objective evaluation of outcomes. Virulence. 2013; 4(2):151-7. DOI: $10.4161 /$ viru. 21626

60. Eljaaly K, Elarabi S. Impact of requiring reauthorization of restricted antibiotics on day 3 of therapy. J Antimicrob Chemother. 2018; 73(2):527-30.

61. Reed EE, Stevenson KB, Jessica E. West, Karri A. 
Bauer, and Debra A. Goff. Impact of formulary restriction with prior authorization by an antimicrobial stewardship program. Virulence 2013; 4(2):158-62. DOI: 10.4161/viru.21657

62. Schweitzer V, Van Werkhoven C, J Rodríguez Baño, J Bielicki, S Harbarth, M Hulscher, B Huttner, J Islam, P Little, C Pulcini, A Savoldi, E Tacconelli, J-F Timsit, $\mathrm{M}$ van Smeden, $\mathrm{M}$ Wolkewitz, M J M Bonten, A S Walker, and M J Llewelyn. Optimizing design of research to evaluate antibiotic stewardship interventions: consensus recommendations of a multinational working group. Clin Microbiol Infect 2020;26(1):41-50. DOI: 10.1016/j.cmi.2019.08.017.

63. Gravatt LAH, Patterson JA, and S. Franzese . Educational Antimicrobial Stewardship Strategies. Curr. treat. options infect. dis 2016; 8(2):84-92.

64. World Health Organization. Regional office for the eastern Mediterranean. Initiating Egypt's antimicrobial resistance national action plan. Available at http://www.emro.who.int/egy/egyptevents/antimicrobial-resistance-national-actionplan.html

65. Afdal A. AMS Case Study - EGYPT National Antimicrobial Stewardship Program. In: (RDU RDU, editor. Ministry of Health, Egypt: Hospital Pharmacy Administration HPA CAPA; 2019.

66. Rehab El-Sokkary, Rania Kishk, Sally Mohy ElDin, Nader Nemr, Nageh Mahrous, Mostafa Alfishawy, Samar Morsi, Wael Abdalla, Mohamed Ahmed, Rehab Tash. Antibiotic Use and Resistance Among Prescribers: Current Status of Knowledge, Attitude, and Practice in Egypt. Infect. Drug Resist 2021:14:1209-1218 DOI: 10.2147/IDR.S299453

67. Mentor Ali Ber Lucien, Michael F. Canarie,Paul E. Kilgore, Gladzdin Jean-Denis, Natael Fénélon, Manise Pierre,Mauricio Cerpa, Gerard A. Joseph, Gina Maki, Marcus J. Zervos, Patrick Dely, Jacques Boncy, Hatim Sati, Ana del Rio, and Pilar Ramon-Pardo. Antibiotics and antimicrobial resistance in the COVID-19 era: Perspective from resource-limited settings. Int J Infect Dis 2021:
104:250-254

DOI:https://doi.org/10.1016/j.ijid.2020.12.087

68. Kerry-Anne Hogan, Mohamed Gazarin, and Julie Lapenskie. Development and Implementation of an Antimicrobial Stewardship Program in a Rural Hospital. Can J Hosp Pharm 2016: 69(5): 403-408 DOI: $10.4212 /$ cjhp.v69i5.1595

69. Majumder MAA, Rahman S, Cohall D, Bharatha A, Singh K, Haque M, and Gittens-St Hilaire M. Antimicrobial Stewardship: Fighting Antimicrobial Resistance and Protecting Global Public Health. Infect Drug Resist.2020; 13: 4713-4738. DOI https://doi.org/10.2147/IDR.S290835 